\title{
87
}

\section{LACTOGENIC IMMUNITY IN TRANSGENIC MICE PRODUCING RECOMBINANT ANTIBODIES NEUTRALIZING CORONAVIRUS}

\author{
J. Castilla, ${ }^{1}$ I. Sola, ${ }^{1}$ B. Pintado, ${ }^{2}$ J. M. Sánchez-Morgado, ${ }^{1}$ and L. Enjuanes ${ }^{1}$ \\ 'Department of Molecular and Cell Biology \\ Centro Nacional de Biotecnología, CSIC \\ Campus Universidad Autónoma, Canto Blanco \\ 28049 Madrid, Spain \\ ${ }^{2}$ Department of Animal Reproduction. INIA \\ Carretera La Coruña km 5.9 \\ 28040 Madrid, Spain
}

\section{ABSTRACT}

Protection against coronavirus infections can be provided by the oral administration of virus neutralizing antibodies. To provide lactogenic immunity, eighteen lines of transgenic mice secreting a recombinant $\operatorname{IgG}_{1}$ monoclonal antibody $\left(\mathrm{rIgG}_{1}\right)$ and ten lines of transgenic mice secreting recombinant IgA monoclonal antibodies (rIgA) neutralizing transmissible gastroenteritis coronavirus (TGEV) into the milk were generated. Genes encoding the light and heavy chains of monoclonal antibody (MAb) 6A.C3 were expressed under the control of regulatory sequences derived from the mouse genomic DNA encoding the whey acidic protein (WAP) and beta-lactoglobulin (BLG), which are highly abundant milk proteins. The MAb 6A.C3 binds to a highly conserved epitope present in coronaviruses of several species. This MAb does not allow the selection of neutralization escaping virus mutants. The antibody was expressed in the milk of transgenic mice with titers of one million as determined by RIA, and neutralized TGEV infectivity by one million fold corresponding to immunoglobulin concentrations of 5 to $6 \mathrm{mg}$ per ml. Matrix attachment regions (MAR) sequences were not essential for $\mathrm{rlgG}_{1}$ transgene expression, but co-microinjection of MAR and antibody genes led to a twenty to ten thousand-fold increase in the antibody titer in $50 \%$ of the $\mathrm{rlgG}_{1}$ transgenic animals generated. Co-microinjection of the genomic BLG gene with rIgA light and heavy chain genes led to the generation of transgenic mice carrying the three transgenes. The highest antibody titers were produced by transgenic mice that had integrated the antibody and BLG genes, al- 
though the number of transgenic animals generated does not allow a definitive conclusion on the enhancing effect of BLG co-integration. Antibody expression levels were transgene copy number independent and integration site dependent. The generation of transgenic animals producing virus neutralizing antibodies in the milk could be a general approach to provide protection against neonatal infections of the enteric tract.

\section{INTRODUCTION}

The mucosal immune system and one of its predominant effectors, immunoglobulin, provide the initial immunologic barriers against most pathogens that invade the body at mucosal surfaces (Mazanec et al., 1996). This is especially true for viruses, since resistance to infection has been frequently correlated with the presence of specific antibody in mucosal secretions. Of particular interest is the lactogenic immunity by which the antibodies in the milk confer immunity to newborns. The oral administration of virus neutralizing MAbs may protect mucosal tissues against viral infections that invade enteric areas (Enjuanes and Van der Zeijst, 1995; Saif and Wesley, 1992).

Transgenic technology now makes possible to express novel proteins in the milk under the control of regulatory sequences controlling the expression of abundant milk proteins such as the whey acidic protein (WAP) and the $\beta$-lactoglobulin (Castilla et al., 1997; Sola et al., 1997). This approach could be extended to the expression of virus neutralizing antibodies in order to provide protection against enteric infections.

The immune response to TGEV has been characterized and full protection against TGEV can be provided by lactogenic immunity. It has also been shown that the passive oral administration of serum elicited by recombinant adenoviruses expressing the spike protein completely protects piglets against virulent virus challenge (Torres et al., 1995). Bottle feeding of newborn animals may target the antibody to epithelial surfaces providing protection against enteric virus infections, but this approach is not practical. Alternatively, transgenic animals secreting virus neutralizing antibodies in their milk during lactation should protect piglets against coronavirus infection. To explore this approach, the expression of a recombinant TGEV neutralizing MAb with a human $\mathrm{IgG}_{1}$ and swine IgA isotypes under the control of regulatory sequences controlling the expression of abundant milk proteins was investigated.

TGEV infects both enteric and respiratory tissues and causes a mortality close to one hundred percent when newborn animals are infected (Enjuanes and Van der Zeijst, 1995; Saif and Wesley, 1992). The major antigenic sites of TGEV involved in the induction of virus neutralizing antibodies are located in the globular portion of the spike (S) protein (Correa et al., 1990; Delmas and Laude, 1990; Gebauer et al., 1991). Investigations by our laboratory into the mechanisms of TGEV neutralization and of antigenic and genetic variability (Sanchez $e t$ al., 1992; Sanchez et al., 1990) have led to the identification of mouse MAb 6A.C3 which neutralized all the TGEV isolates tested, and also TGEV-related coronaviruses which infect at least three animal species: porcine, canine, and feline. This MAb probably binds to an epitope essential for virus replication since no neutralization escape mutants were generated (Gebauer et al., 1991) and has been used in the construction of the transgenic mice.

Transgenic mice were constructed carrying two expression cassettes containing the cDNA sequences encoding the light and heavy chains of a chimeric immunoglobulin. $\mathrm{rIgG}_{1} \mathrm{cDNAs}$ were co-microinjected with MAR sequences that increase transgene expression by flanking the transcription units and isolating transgenes from the negative influences of surrounding regions or by altering chromatin structure (Bonifer et al., 1990). rIgA cDNAs were co-microinjected with gene expression regulatory sequences derived 
from the BLG gene. Transgenic mice that secrete high titer virus neutralizing $\operatorname{rlgG}_{1}$ and rIgA into their milk have been obtained.

\section{MATERIALS AND METHODS}

\subsection{Cells, Viruses, and Antibodies}

Swine testis (ST) cells, COS-1 cells, non secreting murine myeloma Sp2/0, MAb 6A.C3, and S2.1 IgA secreting porcine hybridoma were grown as previously described (Castilla, Sola, and Enjuanes, 1997). TGEV PUR46-MAD strain was grown, purified, and titrated in ST cells as described previously (Jiménez et al., 1986). Antibody titers were determined by radioimmunoassay (RIA) using purified TGEV as the antigen following a reported procedure (Jiménez et al., 1986). Heavy chain titers were determined by a double antibody sandwich (DAS) similar to the RIA (Castilla et al., 1997).

\subsection{Western Blot Analysis}

The procedures for Western blot have been described (Correa et al., 1988). The antiserum used to develop the RIA assays to detect rIgG, RIA (Castilla et al., 1997) was rabbit anti-human IgG (Cappel). To detect rIgA rabbit anti-swine IgA (Bethyl Laboratories, Inc. Texas. USA) was used.

\subsection{Construction of Transgenes}

cDNAs encoding the constant modules of human gamma and kappa chains (Castilla, Sola, and Enjuanes, 1997) were expressed using regulatory sequences from the WAP gene. The two cDNA fragments were blunt ended and inserted separately at the unique EcoRV site of the plasmid pWAP7K (Hennighausen and Sippel, 1982) carrying the WAP gene (Fig. 1A).

cDNAs encoding the constant modules of porcine alpha and kappa chains (Sola et al., 1997) were expressed using a BLG construct pSS1tgXS. To generate the expression cassette pBJ41 (Fig. 1B), an EcoRV cloning site was inserted between exons 1 and 5 of BLG gene. Introns 5 and 6 were removed. The cDNA fragments encoding the chimeric light and heavy chains of rIgA were cloned separately at the unique EcoRV site of the plasmid pBJ41.

\subsection{Functional Analysis of Transgenes}

In order to study whether the resulting plasmids carrying the immunoglobulin genes under the control of WAP and BLG regulatory sequences were functional and hormone inducible, mouse mammary gland epithelial cells ( $\mathrm{HC11}$ ) were used. Plasmids encoding immuneglobulin light and heavy chains under the control of WAP or BLG gene regulatory elements were linearized and were co-transfected into $2 \times 10^{7} \mathrm{HC} 11$ cells by electroporation. Antibody expression levels from transformed $\mathrm{HC} 11$ cells were analyzed in the supernatant by RIA after hormone induction.

\subsection{Generation of Transgenic Mice}

Transgenic mice were generated essentially as described (Castilla et al., 1997; Sola et al., 1997). 
A
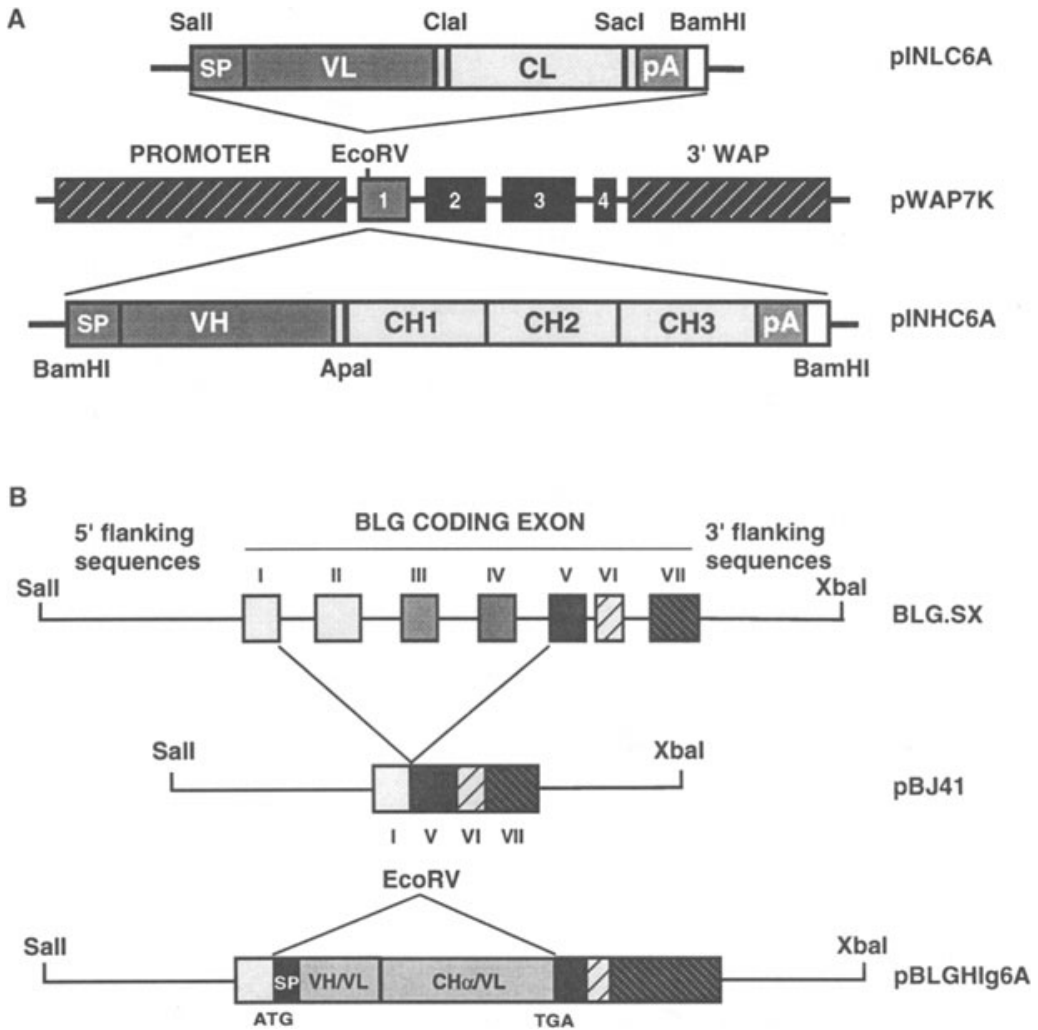

Figure 1. Cloning of immunoglobulin light and heavy chain cDNAs into expression cassettes. (A) The two cDNA fragments encoding the chimeric light and heavy chains of $\mathrm{IgG}_{1}$, were extracted from the plasmids pINLC6A and pINHC6A respectively and inserted separately at the unique EcoRV site of the plasmid pWAP7K carrying the WAP gene. SP, signal peptide. VL and VH, variable modules. $\mathrm{CL}, \mathrm{CH}, \mathrm{CH} 2$ and $\mathrm{CH} 3$, constant modules. pA, SV40 polyadenylation signal. Key restriction endonuclease sites used in the cloning are indicated. (B) Structure of the BLG gene (BLG.SX) indicating the introns deleted to generate the pBJ41 plasmid carrying the intronless BLG-minigene. The ATG present in the BLG was removed in $\mathrm{pBJ} 41$ plasmid and an EcoRV cloning site was created by linkers between exons I and V. Exon VII does not have an open reading frame. CAAT, TATAA and AATAAA regulatory signals are indicated. Bold lines indicate 5'- and 3'-flanking sequences and intronic sequences of BLG; boxes, BLG exon sequences. VH or VL and $\mathrm{CH} \alpha$ or $\mathrm{CL}$, cDNA encoding immunoglobulin chains. All constructs comprise identical 5'- and 3'-regulatory sequences. Relevant restriction enzyme cutting sites are shown. SP, signal peptide.

\subsection{Screening of Founders}

DNA from tail biopsies was prepared as described (Castilla et al., 1997; Sola et al., 1997). The presence of WAP or BLG-immunoglobulin transgenes and MAR sequences were identified by a PCR assay using primers specific for the WAP or BLG promoters, the immunoglobulin variable modules and MAR sequences (Castilla et al., 1997; Sola et al., 1997).

To determine transgene copy number, PCR-positive animals were examined by Southern blot analysis using a probe that detected the expression cassette for the $\mathrm{L}$ and $\mathrm{H}$ 
immunoglobulin genes and also the single copy endogenous WAP gene. Genomic DNA was digested with BglII and three fragments corresponding to endogenous WAP, WAPLC, and WAP-HC genes were detected by hybridization. The radiolabeled DNA fragment used to probe the blot was specific to the common WAP promoter allowing a comparison of the transgene copy number in each animal on the same Southern blot.

In the case of BLG constructs, EcoRI-cleaved DNA was analyzed by agarose gel electrophoresis and Southern blotting. The DNA fragment used to probe the blot was specific for the common BLG promoter and therefore detected the light and heavy immunoglobulin chain genes and the BLG gene simultaneously, allowing the comparison of the copy numbers of the three on the same Southern-blot. Transgene copy number was determined by comparison with known amounts of reference plasmids.

\subsection{Characterization and Functional Activity of the Recombinant Antibody in Mouse Milk}

Milk from four to six month old transgenic mice was collected as described (Castilla et al., 1997).

\section{RESULTS}

\subsection{Engineering TGEV Neutralizing Recombinant Antibodies}

The construction of the chimeric recombinant antibodies required the fusion of MAb 6A.C3 light $(\mathrm{L})$ or heavy $(\mathrm{H})$ variable modules to human or porcine constant modules as previously reported (Castilla et al., 1997; Sola et al., 1997). Immunoglobulin variable modules of the MAb 6A.C3 were cloned by RT-PCR and joined to the constant module of human or porcine antibodies. To verify the functionality of $\mathrm{rIgG}_{1}$ and $\operatorname{rgA}, \mathrm{COS}-1$ and murine Sp2/0 myeloma cells were transiently and stably transformed, respectively, with constructs encoding the chimeric $\mathrm{L}$ and $\mathrm{H}$ chains. Antibody expression levels ranging between 20 to $40 \mu \mathrm{g} / \mathrm{ml}$ were detected in the supernatant. The secreted chimeric immunoglobulins bound TGEV by RIA with titers up to $10^{3}$, and neutralized virus infectivity around $10^{4}$-fold (i.e., neutralization indices, NIs, around 4), indicating that the recombinant immunoglobulins had the expected biological activity.

In order to generate transgenic mice expressing mouse-human $\mathrm{rIgG}_{1} 6 \mathrm{~A} . \mathrm{C} 3$ or the rIgA 6A.C3 in the milk, rMAbs were expressed under the control of WAP or BLG gene regulatory sequences, respectively by inserting immunoglobulin gene cDNAs encoding $\mathrm{L}$ and $\mathrm{H}$ chains of MAb 6A.C3 into exon 1 of the WAP gene (Fig. 1A) or between exons 1 and 5 of a BLG derived expression vector (Fig. 1B).

To study whether antibody expression under the control WAP or BLG regulatory sequences was hormone inducible in epithelial $\mathrm{HC} 11$ cells, these cells were stably transformed with the WAP or BLG-immunoglobulin constructs. Antibody expression was lactogenic hormone dependent (results not shown) indicating that these mouse mammary gland epithelial cells synthesized, assembled and secreted functional recombinant antibodies.

\subsection{Obtention of rIgG $_{1}$ Transgenic Mice}

An equimolar mix of the WAP gene-based expression cassettes encoding $\mathrm{L}$ and $\mathrm{H}$ chains were co-microinjected into the pronucleus of fertilized ova. These expression cas- 
settes were co-microinjected with an equimolar amount of MAR sequences as indicated. To identify founder animals carrying transgenes, genomic DNA isolated from tail biopsies of animals born from microinjected fertilized eggs was screened by PCR for the presence of mouse WAP-immunoglobulin transgenes using primer pairs that hybridized with the WAP gene (5' primer) and with the immunoglobulin gene ( $3^{\prime}$ primer). From embryos microinjected using the immunoglobulin genes without MAR sequences (MAR'), 13 transgenic mice were obtained. From embryos microinjected with the immunoglobulin gene with MAR sequences $\left(\mathrm{MAR}^{+}\right) 33$ transgenic mice were selected. The majority of transgenic mice ( 35 out of 46 ) had co-integrated the transgenes encoding both the light and heavy chains of $\mathrm{rIgG}_{1}$. All 27 animals that were generated from embryos microinjected with DNAs encoding the light and heavy chain of the recombinant antibody with MAR sequences had integrated the three DNAs (data not shown). Transgenic founder animals carrying MAR sequences, light, and heavy genes were bred for further analysis.

A high number of animals $\left(8 \mathrm{MAR}^{-}\right.$and $\left.27 \mathrm{MAR}^{+}\right)$that had integrated both the heavy and light immunoglobulin gene chains transmitted all of these genes to their progeny for at least four generations (data not shown), strongly suggesting that the light and the heavy chains co-integrated in the nineteen selected mice and co-segregated as a single Mendelian character.

\subsection{Construction of rIgA Transgenic Mice}

Analysis of DNA prepared from tail biopsies showed that 23 of the 93 generation zero (G0) mice had integrated at least one of the transgenes. Transgene integration in the genome of a modified animal does not guarantee its expression, since it may be integrated in a silent chromosome region. It has been previously reported that it is possible to enhance the efficiency of transgene expression by co-integrating the expression cassette with a genomic clone of BLG. To attempt an increase of antibody expression, transgenic $\left(\mathrm{BLG}^{+}\right)$mice were produced by co-injection of the BLG gene with the expression cassettes for the light (BLG-SLC) and heavy (BLG-SHC) chains. In all (16 out of 16) of the $\mathrm{BLG}^{+}$transgenic mice in which one or both immunoglobulin genes were integrated, the BLG gene was also integrated (data not shown). The integration of expression cassettes and of genomic BLG was determined by PCR. After screening more than 250 progeny mice, derived from the 16 founder mice, the co-segregation of immunoglobulin and BLG genes was observed in a high proportion of transgenic animals $(>68 \%)$, indicating that in general both immunoglobulin and BLG genes had been integrated in the same chromosomal locus. The majority of the $\mathrm{BLG}^{-}$and $\mathrm{BLG}^{+}$transgenic mice (17 out of 23) had co-integrated the transgenes encoding the light and heavy $\operatorname{rgA}$ chains. Twelve of the $\mathrm{BLG}^{+}$ transgenic mice carrying both light and heavy chains had integrated the BLG gene.

At least 10 out of 17 transgenic founders carrying both SLC+SHC transmitted both transgenes to their progeny suggesting that they have been co-integrated in a single site in each line.

\subsection{Expression of $\mathrm{rIgG}_{1}$ in Milk}

Milk was collected from the generation one (G1) of each transgenic mouse line which transmitted both transgenes. IIgG $_{1}$ was detected by RIA in the milk using TGEV as an antigen. Transgenic $\left(\mathrm{MAR}^{-}\right)$and $\left(\mathrm{MAR}^{+}\right)$produced high titer TGEV specific antibodies in the milk (Fig. 2A). Five out of the five $\mathrm{MAR}^{-}$(two producing the whole antibody and three secreting the heavy chain) secreted TGEV specific antibodies with titers of around $1 \times 10^{2}$ as 

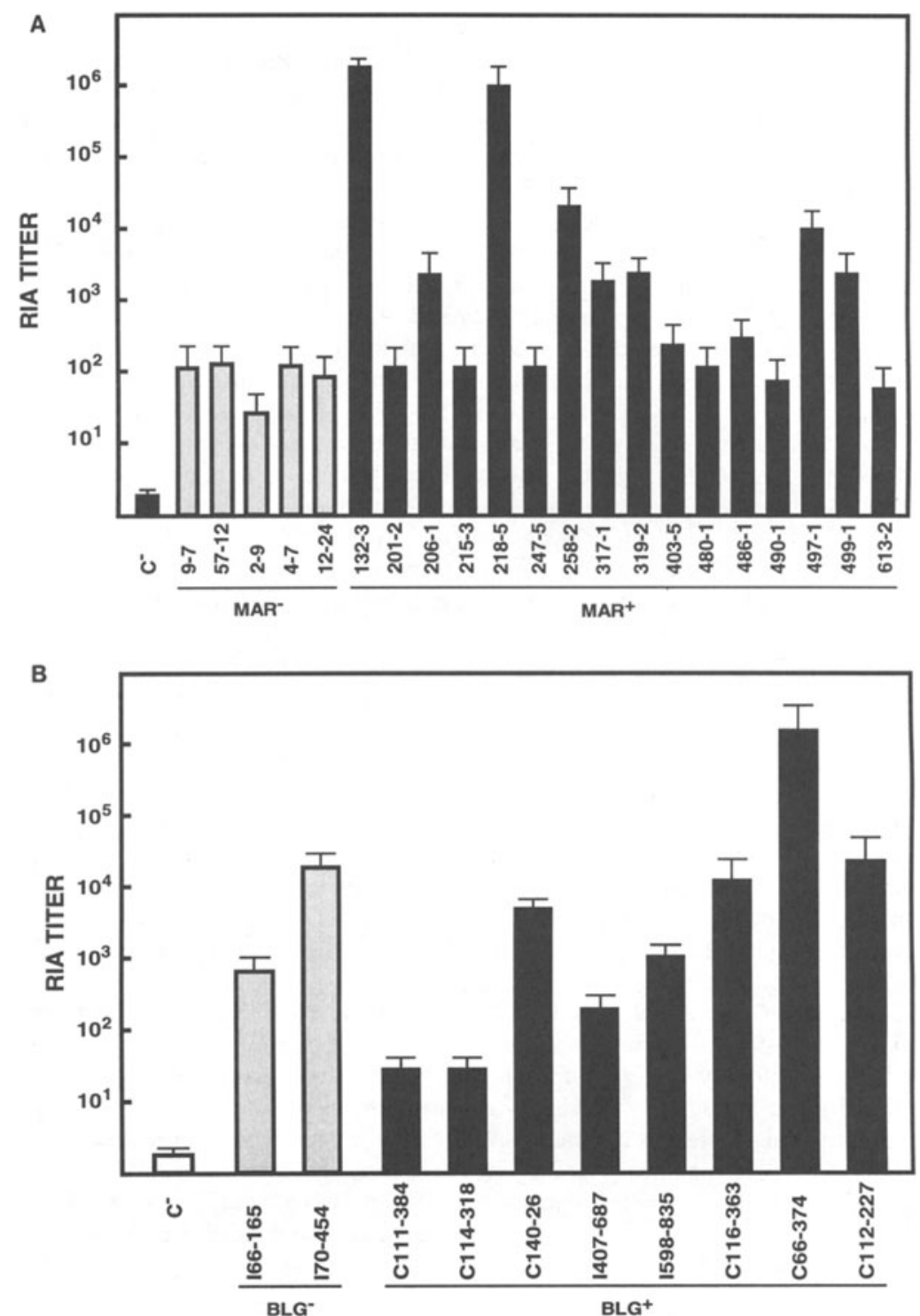

Figure 2. Analysis of recombinant immunoglobulin expression in the milk of transgenic mice. (A) IgG1 expression. Milk from four transgenic mice that were generated by microinjection of immunoglobulin gene expression cassettes without the MAR sequences (MAR $)$ and sixteen mice in which immunoglobulin genes were co-microinjected with MAR sequences $\left(\mathrm{MAR}^{+}\right)$were evaluated for the presence of TGEV binding antibodies by RIA. Four to ten mice of each line were analyzed and the mean \pm the standard deviation of the maximum titers obtained during lactation are shown. $\mathrm{C}^{-}$, milk from non-transgenic mice. (B) IgA expression. The RIA titers for rlgA in the milk of $\mathrm{BLG}^{-}$and $\mathrm{BLG}^{+}$transgenic mice are shown as the mean of the antibody titers in three to six milk samples of the same transgenic mice collected at different days through lactation. $\mathrm{C}^{-}$, milk from non-transgenic mice. 
determined by RIA (Fig. 2A), while 16 out of the 17 transgenic $\mathrm{MAR}^{+}$mice expressed $\mathrm{rIgG}_{1}$ in the milk with titers ranging from $1 \times 10^{2}$ to $1 \times 10^{6}$. Within each line of transgenic mice no significant variation in $\mathrm{rIgG}_{1}$ expression levels was observed. The two MAR mice and the sixteen $\mathrm{MAR}^{+}$transgenic mice that transmitted both light and heavy chain genes to the progeny for at least two generations secreted virus neutralizing antibodies into the milk (results not shown).

The co-integration of MAR sequences was not an essential requirement for the expression of immunoglobulin genes (results not shown). The titers of the secreted TGEV specific antibody or immunoglobulin chain produced in five of the MAR mice were around $10^{2}$. The antibody titers in the milk of these MAR mice were determined by direct RIA in the two lines of transgenic mice secreting both the light and heavy antibody chains, and using a double antibody sandwich (DAS) technique in the three lines of transgenic mice secreting only the heavy chain of the same MAb (Fig. 2A). Interestingly, in the mice that had co-integrated MAR sequences antibody titers in the milk ranged between $10^{2}$ and $10^{6}$. In $50 \%$ of these mice ( 8 out of 16 ) antibody expression levels were increased between twenty and ten thousand-fold in relationship to the antibody levels produced by the MAR mice.

The milk from selected MAR ${ }^{+}$transgenic females 132-3 and 218-5 had TGEV specific antibodies with RIA titers close to $10^{6}$. The milk of these mice showed a reduction in TGEV infectivity around one million-fold (results not shown). These results indicated that the $\mathrm{rIgG}_{1}$ synthesized in the mammary gland and secreted into the milk of transgenic mice was active in TGEV neutralization. Recombinant $\mathrm{IgG}_{1}$ production ranged from $0.005 \mathrm{mg} / \mathrm{ml}$ to approximately $5 \mathrm{mg} / \mathrm{ml}$ in the different mice.

\subsection{Expression of rIgA in Milk}

Milk was collected from G0 females or female progeny of mice which transmitted the transgenes for both the heavy and light genes. Recombinant IgA was detected by RIA in the milk of the two BLG transgenic lines, with titers ranging from $8 \times 10^{2}$ to $3 \times 10^{4}$ (Fig. 2B). Eight out of $12 \mathrm{BLG}^{+}$transgenic founders expressed rIgA in the milk with titers in RIA ranging from $5 \times 10^{1}$ to $5 \times 10^{6}$.

Neutralization assays with milk samples showed that virus infectivity was reduced around one million-fold using milk with the highest titers (mouse C66-374). These results indicated that the rIgA synthesized in the mammary gland and secreted into the milk of transgenic mice was biologically active in TGEV neutralization.

No significant differences in transgene expression frequency were observed between $\mathrm{BLG}^{-}$and $\mathrm{BLG}^{+}$transgenic mice (Fig. $2 \mathrm{~A}$ ). Nevertheless, higher Ab titers $\left(>10^{6}\right)$ were obtained in mice that had cointegrated immunoglobulin and BLG genes (Fig. 2A).

The rIgA concentrations in transgenic mouse milk were measured by RIA using a purified rIgA standard and ranged from $0.005 \mathrm{mg} / \mathrm{ml}$ to $6 \mathrm{mg} / \mathrm{ml}$.

\subsection{Analysis of Transgene Integration}

To determine whether antibody expression levels were transgene copy number dependent, transgenic mice carrying $\mathrm{rIgG}_{1}$ and $\mathrm{rIgA}$ genes were analyzed by Southern blot using a probe specific for a promoter sequence present in the expression cassette for the light and heavy immunoglobulin chain genes and in the endogenous WAP or BLG gene. DNA extracted from the tails of transgenic mice was digested with convenient restriction endonucleases to identify specific fragments (Fig. 3A and $3 \mathrm{C}$ ) which corresponded to the 


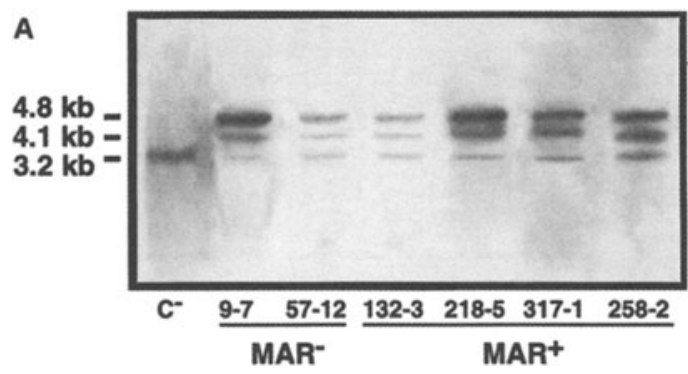

Figure 3. Relationship between transgene copy number and antibody expression levels. (A) Southern blot analysis of WAP-immunoglobulin transgene integration. Genomic DNA from transgenic mice were hybridized with a WAP-specific probe. The bands shown correspond to WAP-heavy transgene $(4.8 \mathrm{~kb})$, WAP-light transgene $(4.1 \mathrm{~kb})$, and endogenous WAP gene ( $3.2 \mathrm{~kb})$ sequences. (B) Antibody expression levels in the milk of transgenic mice with variable numbers of transgene copy numbers. $\mathrm{MAR}^{+}$, with MAR sequences integrated. MAR ${ }^{-}$, without MAR sequences integrated.

\begin{tabular}{|c|c|c|c|c|}
\hline \multirow{2}{*}{ Transg } & \multirow{2}{*}{ enic mice } & \multicolumn{2}{|c|}{ Copy number ${ }^{a}$} & \multirow{2}{*}{ Expression } \\
\hline & & $\begin{array}{l}\text { Light } \\
\text { Chain }\end{array}$ & $\begin{array}{l}\text { Heavy } \\
\text { Chain }\end{array}$ & \\
\hline \multirow[t]{2}{*}{ MAR- $^{-}$} & $9-7$ & 10 & 40 & $1-5 \times 10^{2}$ \\
\hline & $57-12$ & 1 & 3 & $1-5 \times 10^{2}$ \\
\hline \multirow[t]{4}{*}{$\mathrm{MAR}^{+}$} & $132-3$ & 1 & 3 & $1-8 \times 10^{6}$ \\
\hline & $218-5$ & 3 & 12 & $1-5 \times 10^{5}$ \\
\hline & $317-1$ & 2 & 3 & $1-5 \times 10^{3}$ \\
\hline & $258-2$ & 2 & 2 & $1-5 \times 10^{4}$ \\
\hline
\end{tabular}

a. Transgene copy number.

b. Titer, highest dilution giving a binding three-fold higher than the background.

genes encoding the light and heavy immunoglobulin chains and the endogenous genes (WAP and BLG). The intensity of the endogenous gene band was taken as the reference to estimate the integrated immunoglobulin gene copy number. A large variation in the transgene copy number (from 1 to 40 ) was detected in the transgenic lines analyzed (Figure 3B and 3D) and no correlation was observed between antibody expression levels and transgene copy number.

\section{DISCUSSION}

Transmisible gastroenteritis virus produces high mortality in suckling piglets. In order to effectively protect offspring, it is necessary to produce high levels of neutralizing antibodies in the milk of lactating sows. To this end, we have constructed transgenic mice expressing the genes encoding the chimeric TGEV neutralizing MAb 6A.C3 with human constant module ( $\operatorname{IgG}_{1}$ isotype) and porcine constant module (IgA isotype) under the control of abundant milk protein (WAP and BLG) gene regulatory elements, respectively.

Transgenic mice were constructed that secreted the $\mathrm{rIgG}_{1}$ and $\operatorname{rIgA} \mathrm{MAb}$ into their milk with titers up to $5 \times 10^{6}$ as determined both by RIA and neutralization. Chimeric IgA antibodies have been shown to be more efficient in virus neutralization than the recombinant antibodies with identical specificity and the IgG isotype, probably because of the tetravalency of dimeric rIgA.

The expression levels of functional TGEV-specific $\operatorname{rIgG}_{1}$ and $\mathrm{rIgA}$ in the milk of several transgenic mice (up to $6 \mathrm{mg} / \mathrm{ml}$ ) represents one of the highest expression levels ob- 


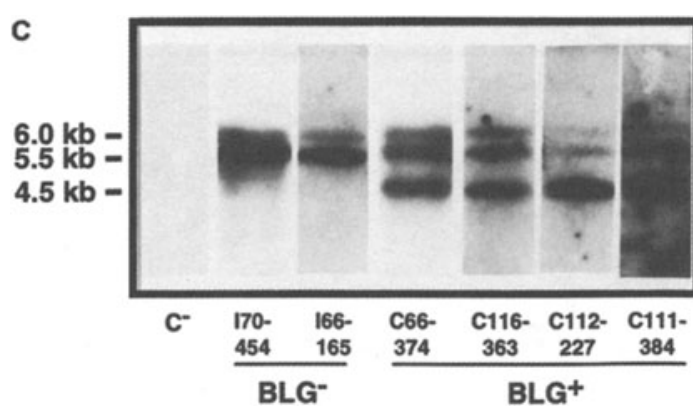

\begin{tabular}{|c|c|c|c|c|}
\hline \multirow{2}{*}{ Transg } & enic mice & \multicolumn{2}{|c|}{ Copy number ${ }^{a}$} & \multirow{2}{*}{ Expression } \\
\hline & enic mice & $\begin{array}{l}\text { Light } \\
\text { Chain }\end{array}$ & $\begin{array}{l}\text { Heavy } \\
\text { Chain }\end{array}$ & \\
\hline \multirow[t]{2}{*}{$\mathrm{BLG}^{-}$} & $170-454$ & 8 & 2 & $3 \times 10^{4}$ \\
\hline & $166-165$ & 6 & 2 & $4-8 \times 10^{2}$ \\
\hline \multirow[t]{4}{*}{$\mathrm{BLG}^{+}$} & C66-374 & 4 & 2 & $1-5 \times 10^{6}$ \\
\hline & $\mathrm{C} 116-363$ & 5 & 3 & $1-8 \times 10^{3}$ \\
\hline & C112-227 & 4 & 2 & $1-5 \times 10^{4}$ \\
\hline & C111-384 & 6 & 2 & 50 \\
\hline
\end{tabular}

a. Transgene copy number.

b. Titer, highest dilution giving a binding three-fold higher than the background.
Figure 3. (Continued) (C) Southernblot analysis of $\mathrm{BLG}^{-}$and $\mathrm{BLG}^{+}$transgenic lines. $\mathrm{C}^{*}$, non-transgenic mouse DNA. Tail DNA samples were cleaved with EcoRI and analyzed by electrophoresis and Southern-blot. Filters were probed with 5' flanking sequences common to the three transgenes. The $6.0 \mathrm{~kb}$ BLG-SHC, $5.5 \mathrm{~kb}$ BLG-SLC and $4.5 \mathrm{~kb}^{\mathrm{BLG}}{ }^{-}$transgene specific EcoRI fragments are shown. (D) Comparison of rIgA titers in $\mathrm{BLG}^{-}$ and $\mathrm{BLG}^{+}$transgenic lines carrying different transgene copy numbers. The expression levels are reported as the RIA titers of the highest dilution giving a binding three-fold the background. The RIA titers of rIgA in the milk of $\mathrm{BLG}^{-}$and $\mathrm{BLG}^{+}$transgenic mice were calculated as the mean of antibody titers of three to six milk samples from the same mouse.

served in a complex recombinant protein in any mammalian expression system, including transgenic mice. This result was particularly interesting since it indicated that the epithelial cells of the mammary gland successfully produced both the light and heavy immunoglobulin chains and provided the adequate environment for the assembly of a functional $\mathrm{IgG}_{1}$ and $\mathrm{IgA}$ molecule.

Recombinant immunoglobulin expression levels in the milk of sows, similar to those produced by the transgenic mice described in this manuscript, may suffice to protect piglets against TGEV infection. The recombinant immunoglobulin that has been used contains the variable modules of the MAb 6AC3, that neutralizes very efficiently all known TGEV strains and does not lead to the selection of neutralization escape mutants. This fact, the continuous intake of virus neutralizing recombinant antibodies from the milk of trangenic sows during lactation and the natural defenses should provide in vivo protection against TGEV infection.

In the experimental model system used, the transgenic lines of mice that were not co-microinjected with MAR sequences also expressed rIgG $_{1}$. Consequently, we could not assert the effect of MAR sequence inclusion on the frequency of antibody-expressing mice. Among the mice that co-integrated MAR sequences with $\mathrm{rIgG}_{1}$ genes, no direct relationship between transgene copy number and the $\mathrm{rIgG}_{1}$ expression levels in the milk was found. This suggests that transgene integration site determines transcriptional levels.

In contrast with the findings of other investigators (Clark et al., 1993), the co-microinjection of BLG sequences with antibody genes has not led to a significant increase of 
the average antibody expression levels, since in the absence or in the presence of BLG gene, aproximately the same antibody titers were obtained in the milk of transgenic mice. Nevertheless, it is interesting to note that maximum antibody expression levels were obtained when BLG and antibody sequences were co-microinjected, although, since a small number of transgenic is presented, the significance of BLG co-integration to achieve high antibody expression levels can not be definitively concluded. The requirement for introns to achieve an efficient transgene expression, probably due to the presence of cis-acting elements, is well documented (Whitelaw et al., 1991). However, in our system, cDNAs encoding rIgA light and heavy chains were inserted into BLG intronless constructs and an efficient expression of rIgA was observed in the milk of the transgenic animals, suggesting that sequences within these cDNAs can also favor expression. One possibility is that some sequences present in the variable module of MAb 6A.C3 light and heavy chains may enhance expression, since this MAb was selected from two thousand independently generated MAbs because its specificity and high expression levels. In this context, transgene rescue by co-microinjecting the BLG gene with the transgenes (Clark et al., 1993; Clark et al., 1992) may not enhance the efficiency of expression as dramatically as in those cases with a very inefficient expression of the intronless transgenes.

Normal development in the mice secreting high titer coronavirus neutralizing antibodies in the milk was observed, indicating that the production of pathogen neutralizing antibodies in the milk could be a useful approach to prevent enteric infections of the newborn.

The modular approach to obtain recombinant antibodies (i.e., the fusion of variable to constant immunoglobulin domains) described in this manuscript could easily be applied to other antibodies with different therapeutic purposes. Transgenic swine expressing TGEV-neutralizing recombinant immunoglobulins are currently being made using the same expression cassettes described in this paper. This new system will allow us to directly test the lactogenic immunity provided by the transgenic sows to neonates following challenge with TGEV. Since MAbs specific for many enteric viruses are available, this strategy could be a general procedure to generate animals resistant to numerous viral infections of the enteric tract.

\section{REFERENCES}

Bonifer, C., Vidal, M., Grosveld, F., and Sippel, A. E., 1990, Tissue specific and protein position independent expression of the complete gene domain for chicken lysozyme in transgenic mice, EMBO J. 9: 2843-2848.

Castilla, J., Pintado, B., Sola, I., Sánchez-Morgado, J. M., Hennighausen, L., and Enjuanes, L., 1998, Engineering lactogenic immunity in transgenic mice secreting virus neutralizing antibodies in the milk, Nat. Biotech. 16: $349-353$.

Castilla, J., Sola, I., and Enjuanes, L., 1997, Interference of coronavirus infection by expression of immunoglobulin $\mathrm{G}(\mathrm{IgG})$ or IgA virus-neutralizing antibodies, J. Virol. 71: 5251-5258.

Clark, A. J., Archibald, A. L., McClenaghan, M., Simons, J. P., Wallace, R., and Whitelaw, C. B. A., 1993, Enhancing the efficiency of transgene expression, Transg. Res. Soc. Lond. 339: 225-232.

Clark, A. J., Cowper, A., Wallace, R., Wright, G., and Simons, J. P., 1992, Rescuing transgene expression by co-integration, Biotechnology 10: 1450-1454.

Correa, I., Gebauer, F., Bullido, M. J., Suñé, C., Baay, M. F. D., Zwaagstra, K. A., Posthumus, W. P. A., Lenstra, J. A., and Enjuanes, L., 1990, Localization of antigenic sites of the E2 glycoprotein of transmissible gastroenteritis coronavirus, J. Gen. Virol. 71: 271-279.

Correa, I., Jiménez, G., Suñé, C., Bullido, M. J., and Enjuanes, L., 1988, Antigenic structure of the E2 glycoprotein from transmissible gastroenteritis coronavirus, Virus. Res. 10: 77-94.

Delmas, B., and Laude, H., 1990, Assembly of coronavirus spike protein into trimers and its role in epitope expression, J. Virol. 64: 5367-5375. 
Enjuanes, L., and Van der Zeijst, B. A. M., 1995, Molecular basis of transmissible gastroenteritis coronavirus epidemiology. In "The Coronaviridae" (S. G. Siddell, Ed.), pp. 337-376. Plenum Press, New York.

Gebauer, F., Posthumus, W. A. P., Correa, I., Suñé, C., Sánchez, C. M., Smerdou, C., Lenstra, J. A., Meloen, R., and Enjuanes, L., 1991, Residues involved in the formation of the antigenic sites of the $\mathrm{S}$ protein of transmissible gastroenteritis coronavirus, Virology 183: 225-238.

Hennighausen, L. G., and Sippel, A. E., 1982, Mouse whey acidic protein is a novel member of the family of fourdisulfide core proteins, Nuc. Acid. Res. 10: 2677-2684.

Jiménez, G., Correa, I., Melgosa, M. P., Bullido, M. J., and Enjuanes, L., 1986, Critical epitopes in transmissible gastroenteritis virus neutralization, J. Virol. 60: 131-139.

Mazanec, M. B., Huang, Y. T., Pimplikar, S. W., and Lamm, M. E., 1996, Mechanisms of inactivation of respiratory viruses by IgA, including intraepithelial neutralization, Sem. Virol. 7: 285-292.

Saif, L. J., and Wesley, R. D., 1992, Transmissible gastroenteritis. Seventh ed. In "Diseases of Swine" (A. D. Leman, B. E. Straw, W. L. Mengeling, S. D'Allaire, and D. J. Taylor, Eds.), pp. 362-386. Wolfe Publishing Ltd, Ames. Iowa.

Sanchez, C. M., Gebauer, F., Suñé, C., Méndez, A., Dopazo, J., and Enjuanes, L., 1992, Genetic evolution and tropism of transmissible gastroenteritis coronaviruses, Virology 190: 92-105.

Sanchez, C. M., Jiménez, G., Laviada, M. D., Correa, I., Suñé, C., Bullido, M. J., Gebauer, F., Smerdou, C., Callebaut, P., Escribano, J. M., and Enjuanes, L., 1990, Antigenic homology among coronaviruses related to transmissible gastroenteritis virus, Virology 174: 410-417.

Sola, I., Castilla, J., Pintado, B., Sánchez-Morgado, J. M., Whitelaw, B., Clark, J., and Enjuanes, L., 1998, Transgenic mice secreting coronavirus neutralizing antibodies into the milk, J. Virol. 72: 3762-3772.

Torres, J. M., Sánchez, C. M., Suñé, C., Smerdou, C., Prevec, L., Graham, F., and Enjuanes, L., 1995, Induction of antibodies protecting against transmissible gastroenteritis coronavirus (TGEV) by recombinant adenovirus expressing TGEV spike protein, Virology 213: 503-516.

Whitelaw, C. B. A., Archibald, A. L., Harris, S., McClenaghan, M., Simons, L. P., and Clark, A. J., 1991, Targeting expression to the mammary gland: intronic sequences can enhance the efficiency of gene expression in transgenic mice, Transgenic Res. 1: 3-13. 